\title{
Simulating Electromagnetic Field inside Small Aircraft from Wireless Camera Equipment
}

\author{
Clifford De Raffaele, Carl J. Debono and Adrian Muscat \\ Department of Communications and Computer Engineering \\ University of Malta, MSD 2080 \\ Malta \\ cderaffaele@ieee.org, c.debono@ieee.org, adrian.muscat@um.edu.mt
}

\begin{abstract}
The proliferation of wireless systems has urged the aviation industry to consider the exploitation of such a technology to introduce new services and to curtail its cabling weight requirements. Nevertheless, prior to implementing such communication systems, manufactures must ensure that the paramount criterion of aircraft safety is unaffected and thus the potential electromagnetic (EM) field posed on aircraft equipment must be carefully assessed and respective countermeasures taken. To this end, this paper adopts a frequency-domain based threedimensional ray tracing algorithm, based on geometrical optics to compute the EM field generated from the use of wireless camera equipment transmitting at $2.4 \mathrm{GHz}$ inside the cabin of a typical business jet. The model comprehensively considers the various propagation methodologies utilized by multipath signals to provide a holistic understanding of the resultant average field strength incident at each location inside the fuselage.
\end{abstract}

Keywords-electromagnetic interference; ray-tracing; wireless propagation

\section{INTRODUCTION}

The electromagnetic spectrum has witness unprecedented utilization in the past years, with an ever-increasing number of communication systems sharing the same resource [1]. The augmentation in availability of wireless services has prompted engineers to look into the possibility of employing such systems to provide added in-flight services and to curtail the amount of cabling implemented in aircraft. Nevertheless, the adaptation of wireless communication inside aircraft is not straight forward, as the available technologies pose critical electromagnetic interference (EMI) issues [2]. This is evermore so, since safety of a commercial aircraft can only be guaranteed whilst preserving the integrity of the aircraft's communications and control equipment [3]. The past years have thus attested escalated concerns for the penetration of high-intensity radiated fields (HIRF) into conducting enclosures, particularly after a number of aviation incidents have been attributed to the disruption of communications or loss of accuracy of the navigation equipment [4].

Apart from the occurrence of HIRF interference incident from external sources into the aircraft, EMI generated from onboard systems is also a paramount issue for concern [5]. The latter category of EM fields is caused by a repertoire of personal and structural devices which operate at several distinct frequencies in the mobile communications domain.
Furthermore, the diversity and quantity of these peripherals onboard aircraft is expected to proliferate since both industry and academia alike are pushing the boundary for seamless connectivity to a variety of services during flight [6][7].

Providing information by means of natural propagation in the hostile wireless environment is however a complex task for communications. The incoming signals at a receiver are usually derived from a number of distinct paths, all undergoing different attenuation phenomena due to reflections, refractions, diffractions and scattering within the transmission media [8]. Consequently, the need for characterization of field strength and interference in both outdoor and indoor environments has been heavily discussed in literature with several diverse approaches being described.

Estimating the electromagnetic field level in indoor environments is significantly more complex than its external counterpart, as the electric field is composed from a substantially larger number of relevant indirect components [9]. Theoretical models, based directly on the principles of physics and Maxwell's Equations, such as Finite-Difference Time-Domain (FDTD) [10] and the Method-of-Moments [11], provide considerably accurate results for EM field calculation. Unfortunately however, they fail to scale up well for feasible implementation in scenarios concerning large objects. Nonetheless, the rapid advancements in processing simulation capabilities have reinforced the recognition of Ray-Tracing techniques for the characterization of frequency-dependent EM propagation in large scale static environments [12][13].

The peculiar environment of an aircraft structure presents a number of unique characteristics and challenges for the calculation of EMI, which make conventional ray-tracing algorithms inappropriate. The dense cluttering of furniture inside the aircraft cabin significantly inhibits direct propagation, whilst on the other hand, the conductive tunnellike structure of the fuselage presents waveguide effects for EM signals [14].

To this end, this paper focuses on the application of a developed three dimensional ray-tracing algorithm to model EM fields inside the cabin of a typical business jet generated by wireless camera equipment transmitting inside the aircraft and is organized as follows; Section II outlines the ray tracing technique used, together with a delineation of the propagation parameters employed. The implementation of a simulation 
scenario is presented in Section III together with the results obtained. Finally, a conclusion is carried out in Section IV.

\section{THE RAY-TRACING TECHNIQUE}

Evaluation of the EMI field incident on the inner fuselage structure and in particulate on the avionics panel is executed by implementing an area oriented design based on ray launching techniques. The computational complexity of the developed algorithm is quasi-independent on the number of observation points examined whilst linearly dependent on the amount of subsequent environment interactions [15]. The developed framework invokes the use of the Geometric Optics (GO) hypothesis since the dimensions of structures within the aircraft environment are much smaller than the wavelength of the communication signals considered. Thus, the average EM field can be considered as the combined contribution of distinct rays and the calculation of each propagation mechanism can be done separately as described by [14]:

$$
P_{k}=P_{T}\left(\frac{\lambda}{4 \pi r}\right)^{2} \prod_{i} \rho_{i} \prod_{j} \tau_{j}
$$

where $P_{T}$ is the power transmitted in Watts at wavelength $\lambda$, $r$ is the unfolded path length in meters, while $\rho_{i}$ and $\tau_{j}$ represent the contribution of reflection and transmission coefficients of multipath rays. Transmitting nodes in the framework are considered to be stationary and transmitting at pre-defined power levels in all relevant directions. The latter is achieved by launching rays in directions determined by a Monte-Carlo random approach using one-dimensional probability density functions on the angular components of spherical coordinates. This ensures that holistic coverage is achieved from the antenna and results are emulated in an asymptotical manner.

EM propagation is calculated by modeling each ray with a wavefront perpendicular to its direction of travel. To account for different polarization effects experienced by each ray as it propagates through the media, the former is separated into parallel and perpendicular components, denoted respectively by $\|$ and $\perp$. During simulation, the rays are propagated through the media whilst taking into account the path loss that each ray experiences while traveling on the pathway according to Friis calculations.

Once a ray wavefront impinges on an object in the cabin, represented as a boundary of media discontinuity, the power of the incident ray is considered for the computation of Fresnel's equations [16]:

$$
\begin{gathered}
R_{\perp}=\frac{\mu_{t} \eta_{i} \cos \theta_{i}-\mu_{i} \eta_{t} \cos \theta_{t}}{\mu_{t} \eta_{i} \cos \theta_{i}+\mu_{i} \eta_{t} \cos \theta_{t}} A_{\perp}, \\
R_{\|}=\frac{-\mu_{i} \eta_{t} \cos \theta_{i}+\mu_{t} \eta_{i} \cos \theta_{t}}{\mu_{i} \eta_{t} \cos \theta_{i}+\mu_{t} \eta_{i} \cos \theta_{t}} A_{\|},
\end{gathered}
$$

$$
T_{\perp}=\frac{2 \mu_{t} \eta_{i} \cos \theta_{i}}{\mu_{t} \eta_{i} \cos \theta_{i}+\mu_{i} \eta_{t} \cos \theta_{t}} A_{\perp},
$$

$$
T_{\|}=\frac{2 \mu_{t} \eta_{i} \cos \theta_{i}}{\mu_{i} \eta_{t} \cos \theta_{i}+\mu_{t} \eta_{i} \cos \theta_{t}} A_{\|} .
$$

These equations, derived from Maxwell's deductions, compute the ratio of power of the reflected $R$ and transmitted $T$ rays as a product of the incident ray $A$ at angle $\theta$, by taking into account the electromagnetic properties $\mu$ of the boundary materials, together with their complex refractive index $\eta$ defined by:

$$
\eta=\sqrt{\mu \varepsilon}+i \frac{c}{\omega \delta}
$$

where $\varepsilon$ represents the permittivity of the medium, $\omega$ is the angular frequency, and $\delta$ describes the skin depth of the propagation material.

EM calculation accuracy is augmented inside the proposed framework by modelling the structural components inside the cabin with their actual 3D volume. Thus, transmitted rays inside the thickness of the object media are considered to experience attenuation proportional to the permittivity, electrical conductivity, and propagated distance until the ray is incident on the opposite boundary of the fixtures. Such a computation conforms with the principals of thin film optics which result in a scenario whereby a residue of the transmitted ray continues to propagate inside a parallel bounded medium due to reflected components as illustrated in Fig. 1.

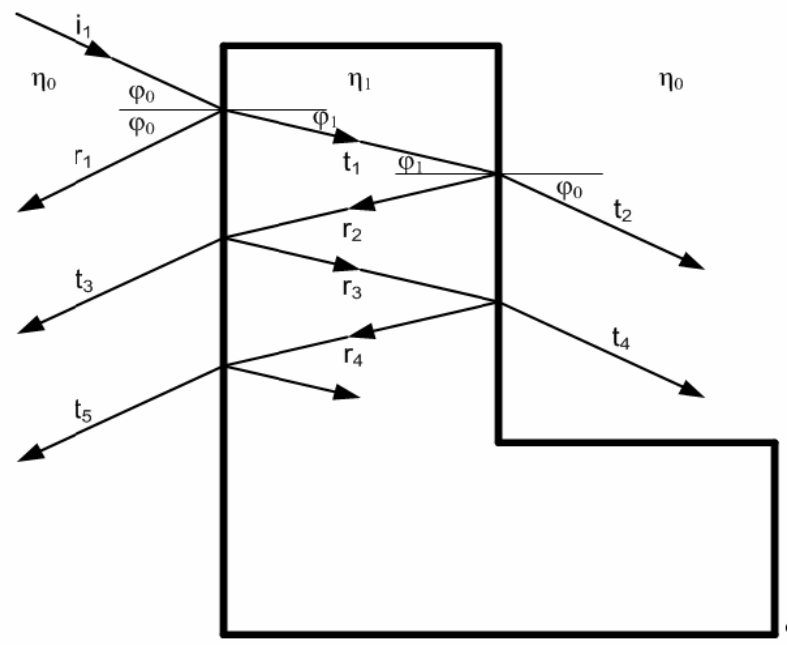

Figure 1. Propagation of rays inside cabin seating modeled as thick media bounded by the air medium.

The reflected ray off the boundary is subsequently also inferred a phase shift of $\pi$ so as to truthfully account for the multipath interference caused by the same ray [17]. The directions of propagation by which the transmitted and 
reflected rays travel following incidence are defined from three-dimensional vector mathematics in the equations [18]:

$$
R=2(N \cdot I) N-I
$$

$$
T=\left(\frac{\eta_{i}}{\eta_{t}} N \cdot I-\sqrt{1-\frac{\eta_{i}^{2}}{\eta_{t}^{2}}\left[1-(N \cdot I)^{2}\right]}\right) N-\frac{\eta_{i}}{\eta_{t}} I,
$$

where $I$ represents incident vector on the boundary, $N$ is the $3 \mathrm{D}$ vector normal to the plane and the vectorial dot product calculation is referred to by the operator $(\cdot)$.

To further enhance and improve the validity of the algorithm framework, the fact that materials are composed of irregular surfaces is taken into account [19]. The algorithm thus computes the specular reflection at each boundary impingement by considering the variances in height of material surfaces that exceed Raleigh's criterion.

\section{SiMULATION AND RESULTS}

This paper considers the implementation of the proposed algorithmic framework, validated in the previous contribution [14], to calculate the EM field incident on the internal fuselage cabin of a typical small business aircraft. The model geometry is produced in three dimensions at a spatial resolution of $5 \mathrm{~cm}$ using the Matlab ${ }^{\circledR}$ platform. As illustrated in Fig. 2, the passenger aircraft is mainly composed of an aluminum fuselage with acrylic window panes. The interior cabin is furnished with wooden flooring and rows of leather seats providing a ten passenger configuration. Prior to simulation, each material was assigned respective electromagnetic parameters, tabulated in Table 1, in order to compute the complex coefficients of the medium.

TABLE I

Electromagnetic MATERIAL PROPERTIES

\begin{tabular}{|l|l|l|l|l|}
\hline \multirow{2}{*}{ Parameters } & \multicolumn{3}{|l|}{ Material } \\
\cline { 2 - 5 } & Air & Seat & Wood & Aluminum \\
\hline Permittivity $(\varepsilon)$ & 3 & 3 & 3 & 1.0005 \\
\hline Permeability $(\mu)$ & 1 & 1 & 1 & 1 \\
\hline $\begin{array}{l}\text { Electrical } \\
\text { Conductivity }(\sigma)\end{array}$ & $5 \mathrm{e}^{-15}$ & 0.01 & 0.01 & $4 \mathrm{e}^{7}$ \\
\hline
\end{tabular}

The simulation performed considers the implementation of three wireless $2.4 \mathrm{GHz}$ cameras that are fitted on the exterior and interior of the aircraft as depicted in Fig. 2. The exterior cameras are typically found in these positions in commercial aircraft to enhance the in-flight entertainment experience. The interior one is seen for surveillance purposes. This configuration would curtail the cabling weight necessary inside the aircraft to obtain such video data. The EM field was simulated by considering 200,000 distinct rays emanating sequentially from each antenna in all directions. Each ray is emitted at an equivalent isotropically radiated power (EIRP) of $20 \mathrm{dBm}$ and simulated until the signal strength goes below $-150 \mathrm{dBm}$, at which point the contribution to the total EMI field is negligible [20].

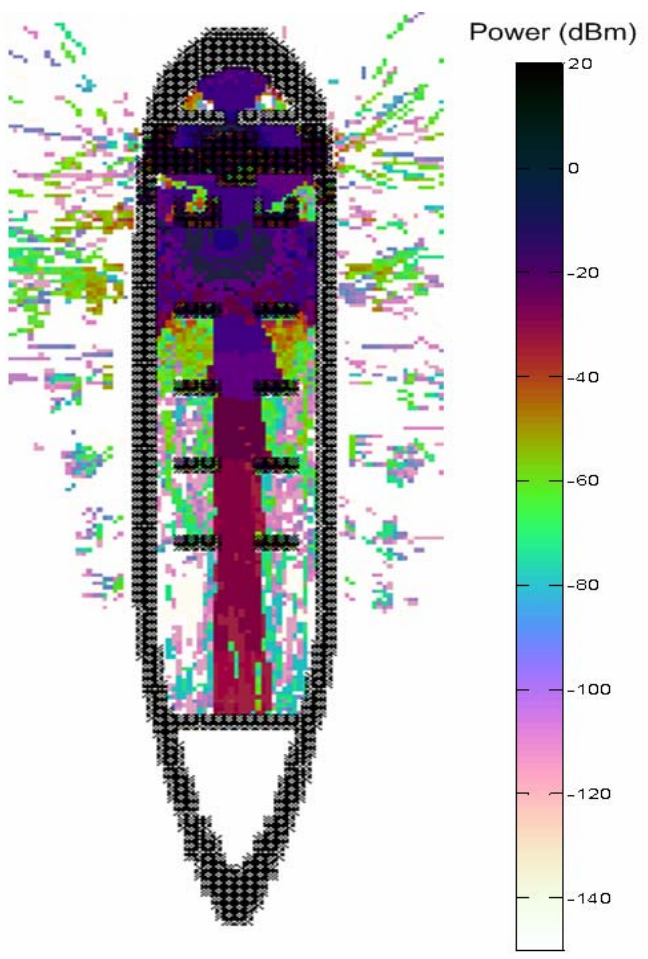

Figure 3. Resultant EM propagation map incident on the internal fuselage by means of the three wireless cameras at $2.4 \mathrm{GHz}$.

The simulation results illustrated in Fig. 3 show that although the power delivered by each wireless antenna is heavily attenuated once it impinges on a furniture structure. The various multipath effects of the clustered configuration are still able to provide substantial coverage throughout the

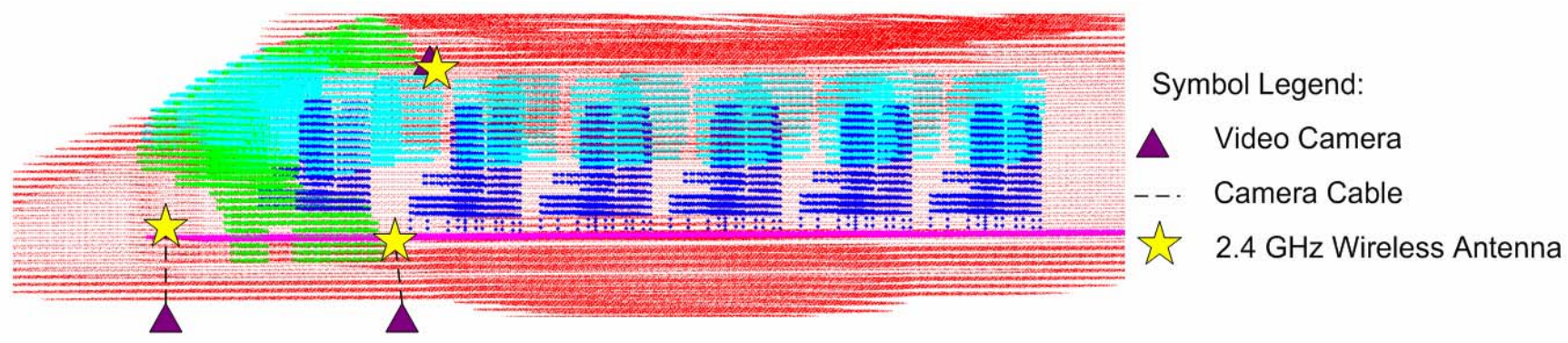

Figure 2. Simulated geometry model of a typical small business jet equipped with an installation of three wireless cameras. 
aircraft's cabin which ensures that each passenger receives a consistent signal. Unfortunately however, the particular location of the camera locations, which are designed to provide the aircraft passengers and crew with monitoring capabilities during landing maneuvers, implies that these are situated at the frontal part of the fuselage. Thus, due to the inherent proximity to the cockpit area, the EM field incident on the instrumentation panel must be taken into consideration. The simulation framework thus exposed the resultant EM field incident on the latter at $2.4 \mathrm{GHz}$ in Fig. 4. The results attained from this representation testify that appropriate consideration must be provided during the design and installation of the network since at the provided configuration, the instrumentation panel is subjected to an average power of $-11.352 \mathrm{dBm}$ with peak locations receiving an illumination power of $-6.49 \mathrm{dBm}$.

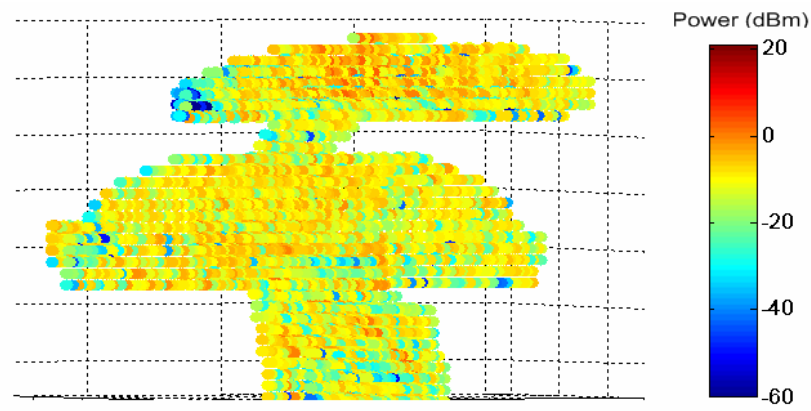

Figure 4. Simulation results for the EM field incident on the instrumentation panel present in the business jet's cockpit.

The significant data obtained from this comprehensive simulation by the EM map highlight the compelling need for the development of better cable shielding. This is even more critical for the cables and systems employed for communications and control of the aircraft. The map obtained allows the identification of areas where cables can be safely routed in internal cabin regions experiencing low EMI, thus mitigating possible interference.

\section{CONCLUSION}

This paper presented the implementation of a frequencybased, ray-tracing algorithm to calculate the EM field incident on the aircraft cabin from the installation of three wireless video cameras. The computed EM signals take into consideration the various multipath effects occurring as the wireless signals propagate inside the fuselage. The study also focused on the EMI field incident on the aircraft instrumentation panel to provide an all-encompassing understanding of the resulting EM effects, whilst offering support for the conscientious development of wireless network design inside aircraft.

\section{ACKNOWLEDGMENT}

This work forms part of the project HIRF SE which is financially supported under the European Union 7th Framework Programme (FP7). The authors are solely responsible for the contents of the paper which does not represent the opinion of the European Commission.

\section{REFERENCES}

[1] M.C. Lawton and J.P. McGeehan, "The application of GTD and ray launching techniques to channel modeling for cordless radio systems," in Proc. of 42nd IEEE Vehicular Technology Conf., pp. 125-130, Denver, 1992.

[2] C. De Raffaele, C.J. Debono and A. Muscat, "Modeling electromagnetic interference generated by a WLAN system onboard commercial aircraft," in Proc. of the 15th IEEE Mediterranean Electrotechnical Conference, pp. 699-704, Malta, Apr. 2010.

[3] K.W. Hurst and S.W. Ellingson, "Path loss from a transmitter inside an aircraft cabin to an exterior fuselage-mounted algorithm," in IEEE Trans. on Electromagnetic Compatibility, vol. 50, no. 3, pp. 504-512, August 2008.

[4] S.V. Georgakopoulos, C.R Birtcher, C.A. Balanis and R.A. Renaut, "HIRF penetration and PED coupling analysis for scaled fuselage models using a hybrid subgrid $\operatorname{FDTD}(2,2) / \operatorname{FDTD}(2,4)$ method," in IEEE Trans. on Electromagnetic Compatibility, vol. 45, no. 2, pp. 293-305, May 2003.

[5] 2001 Federal Radionavigation Systems, DOT-VNTSC-RSPA0103.1/DOD-4650.5, U.S. Department of Defence and Transportation, Washington, DC, 2001.

[6] A. Jahn, et. al. "Evolution of aeronautical communications for personal and multimedia services," in IEEE Communications Magazine, Vol. 41, pp. 36-43, July 2003.

[7] M. Werner and M. Holzbock, "System design for aeronautical broadband satellite communications", in Proc. of IEEE International Conference on Communications, pp.2994-2998, May 2002.

[8] A. Neskovic, N. Neskovic and G. Paunovic, "Modern approaches in modeling of mobile radio systems propagation environment," in IEEE Communications Surveys, pp. 2-12, third quarter, 2000.

[9] A. Kaouris, M. Zaras, M. Revithi, N. Moraitis and P. Constantinou, "Propagation measurements inside a B737 aircraft for in-cabin wireless networks," in Proc. of IEEE Vehicular Technology Conf., pp. 29322936, Singapore, May 2008.

[10] A. Taflove, Computational Electrodynamics, The Finite-Difference Time-Domain Method, Artech House, Boston, 1995.

[11] R.F. Harrington, Field Computation by Moment Methods, Macmillan, New York, 1968.

[12] R. Mittra, Numerical and Asymptotic Techniques in Electromagnetics, Springer Verlag, New York, 1975.

[13] K.H. Ng, E.K. Tameh, A. Doufexi, M. Hunukumbure and A.R. Nix, "Efficient multielement ray tracing with site-specific comparisons using measured MIMO channel data," in IEEE Trans. on Vehicular Technology, vol. 56, no. 3, pp. 1019-1032, May 2007.

[14] C. De Raffaele, C.J. Debono and A. Muscat, "Small aircraft cockpit electromagnetic interference due to UMTS signal propagation", in Proc. of IEEE Int. Conf. on Wireless Information Technology and Systems, Honolulu, Hawaii, Aug. 2010.

[15] E. Geschwendtner, G. Wolfle, B. Burk and F.M. Landstrofer, "Ray tracing vs. ray launching in 3-D microcell modeling" in Proc. of the European Personal and Mobile Communications Conf., pp 74-79, 1995.

[16] D. Didascalou, "Ray-optical wave propagation modelling in arbitrarily shaped tunnels", Ph.D. dissertation, Univ. of Karlsruhe, Germany, 2000.

[17] M. Born and E. Wolf, Principles of Optics, 7th Ed., Cambridge University Press, Cambridge, 1999.

[18] E. Lengyel, Mathematics for 3D Game Programming and Computer Graphics, 2nd Ed., Charles River Media, 2003.

[19] O. Landron, M.J. Feuerstein and T.S. Rappaport, "In situ microwave reflection coefficient measurements for smooth and rough exterior wall surfaces", in IEEE Trans. in Vehicular Technology, Vol. 51, No. 4, pp. 1251-1258, July 2005.

[20] C. De Raffaele, C.J. Debono, A. Muscat, "Modeling electromagnetic signal levels falling on aircraft from satellite communication systems," in Proc. of the 2011 IEEE Aerospace Conference, Montana, USA, March 2011. 